\title{
Review of: "Honey bee (Apis mellifera) colonies benefit from grassland/ pasture while bumble bee (Bombus impatiens) colonies in the same landscapes benefit from non-corn/soybean cropland"
}

Chunsheng $\mathrm{Hou}^{1}$

1 Chinese Academy of Agricultural Sciences

Potential competing interests: The author(s) declared that no potential competing interests exist.

Quinlan et al. entitled "Honey bee (Apis mellifera) colonies benefit from grassland/pasture while bumble bee (Bombus impatiens) colonies in the same landscapes benefit from non-corn/soybean cropland" reported the effects of diverse agricultural land on the different bee species. This study provided a new insight into the species-specific land use but it has to make revision before it is be published.

Major revision

1. In method, authors did not tell how to measure the weight of colonies?

2. In results, authors just show the weight of colonies and lack of the health situation such as behavior, foraging or lifespan.

Minor revision

1. The style of references were not the same. For example, the reference 3 and 4 was different in Journal of economic entomology. 